\title{
Predictions of Transport Accident Fires Using Coupled Structural Dynamics and Computational Fluid Dynamics
}

\author{
ALEXANDER L. BROWN ${ }^{1}$, KURT E. METZINGER ${ }^{2}$, and GREGORY J. WAGNER ${ }^{3}$ \\ Sandia National Labs \\ ${ }^{1}$ Fire and Aerosol Science Department \\ ${ }^{2}$ Solid Mechanics Department \\ ${ }^{3}$ Thermal/Fluid Science \& Engineering Department \\ ${ }^{12} \mathrm{PO}$ Box 5800 \\ Mail Stop 1135 \\ Albuquerque, NM 87185-1135 \\ ${ }^{3} \mathrm{PO}$ Box 969 \\ Mail Stop 9409 \\ Livermore, CA 94551-0969
}

\begin{abstract}
A continuing concern involves transportation accident fires involving liquid fuel vehicles. These can be highly energetic, with the most significant sources of energy typically being the energy of motion (kinetic energy) and the chemical energy in the fuel (fire combustion energy). Recent work has focused on coupling a transient structural dynamics code to a fire computational fluid dynamics code to be able to predict the outcome of such an event. New methods have been developed to permit conservation of mass and momentum between the codes. Other coupling approaches have been developed and are described. This capability has been used to simulate three scenarios. Results from the various test cases suggest discretization accuracy for problems of this class. Validation efforts suggest the accuracy of the methodology, and provide confidence in the predictive capability. These cases also help provide guidance for future application of these methods.
\end{abstract}

KEYWORDS: CFD, fluid dynamics, transportation fires, structural response

\section{NOMENCLATURE LISTING}

$\begin{array}{ll}A & \text { magnitude of undamped oscillations } \\ B & \text { dimensionless separation distance } \\ C & \text { dimensionless TAB model constants } \\ d & \text { diameter (m) } \\ K & \text { new diameter constant } \\ m & \text { mass }(\mathrm{kg}) \\ r & \text { radius (m) } \\ t & \text { time }(\mathrm{s}) \\ u & \text { velocity (m/s) } \\ W e & \text { dimensionless Weber number } \\ y & \text { dimensionless deformation parameter }\end{array}$

$\begin{array}{ll}\text { Greek } & \\ \sigma & \text { surface tension }(\mathrm{N} / \mathrm{m}) \\ \mu & \text { viscosity }(\mathrm{kg} / \mathrm{ms}) \\ \rho & \text { density }\left(\mathrm{kg} / \mathrm{m}^{3}\right) \\ \tau & \text { viscous damping time }(\mathrm{s}) \\ \omega & \text { oscillation frequency }(1 / \mathrm{s}) \\ \text { Subscripts } \\ b & \text { break-up } \\ c r & \text { critical } \\ g & \text { gas } \\ l & \text { liquid } \\ \text { min } & \text { minimum } \\ p & \text { particle } \\ \perp & \text { perpendicular }\end{array}$

\section{INTRODUCTION}

There is a class of transportation accidents that involve significant deformation of the vehicle and release of fuel. These include aircraft impacts and high-speed automotive crashes. They are characterized by a short (order milliseconds to seconds) structural deformation, followed by the release of the liquid fuel which spreads and ignites. This type of incident might be termed a 'combined environment' because it involves multi-disciplinary approach to solve the full sequence of insult. In such cases, one must resolve mechanical deformations and the subsequent fluid spread, fire, and thermal response to predict damage. The ability to 
predict this kind of damage is a relevant engineering problem because an accurate capability can aid in safety analysis, test design, and forensic study.

While there are many applications capable of predicting individual aspects of this problem (i.e., the fluid mechanics alone, the structural dynamics alone), there is little found in the literature on the problem as a whole. Structural/fluid coupling is commonly found for aerospace applications with deforming bodies, but this class of structural modeling is generally not capable of predicting the significant deformations and liquid transport. There is therefore a need for methodology development to model this class of application.

There is an intuitive approach to solving the problem with a structural and a fluid code. One might employ a structural dynamics code to predict the short time scale structural problem, and then use a methodology and the insight gained from that analysis to define the appropriate initial conditions for a subsequent fire analysis requiring longer time scales. This is a general approach we took in defining a capability for this type of analysis. Sandia National Labs has been actively developing simulation tools to model individual aspects of this problem. The codes are developed under a common architecture (Sierra [1]) to facilitate multi-physics coupling. The accuracy of the prediction is then dependent on the accuracy of the individual models and the coupling methods.

Historical work from other research institutions document fire and liquid spread from this class of problem. These include the final report of the World Trade Center fire [2] and the experimental seeding of particles in a fire code[3]. However, they do not directly couple the impact dynamics to the longer-term fire prediction codes. Several reports have described the one-way coupling methods used in this paper in the context of individual scenarios [4-7]. The method involves initializing the fluid mechanic based on solid mechanics calculations. This paper reviews the methods and results from these other reports and summarizes the findings. As there has been an evolution in methods from the first described simulations, this paper describes the methods currently employed based on lessons learned from experience with the method. This paper reviews the previous results, but also focuses on the numerical approximations and the role of the previous studies in the currently recommended methods.

\section{METHODS}

Several developments have been required to make predictions possible using a coupled methodology. The structural and fluid codes alone are advanced simulation codes designed to model physics in their design application space. Particle break-up requires a drop break-up model that is stable and accurate. The code coupling requires a methodology that is stable and is as physically representative of the problem as possible. Even with these tools, there are still issues. The methods section of this paper ends with an analysis of the missing physics in the problem as presently simulated. This discussion helps elucidate the potential improvements to the methods that should be considered to improve method accuracy.

\section{Structural Code}

Presto is a Lagrangian, three-dimensional explicit transient dynamics code built with the Sierra architecture. It is designed to solve structural problems with large deformations and short time scales. Its element library includes smoothed particle hydrodynamics (SPH) elements. This gridless technique can be used to model extremely large deformations without suffering from some of the mesh distortion issues common to traditional finite element formulations. There are also numerous constitutive models, such as the Mie-Gruneisen model, which can be used to model fluids.

SPH is well suited to represent the inertial effects of fluids. However, many aspects of fluid behavior cannot be captured with Presto simulations. The viscous dissipation of the fluid is indirectly modeled through analog properties, and surface tension is not explicitly modeled.

\section{Fluid Code}

Initially, the Presto structural dynamics simulations were coupled to the Vulcan fire code, an off-shoot of the ComputIT Kameleon code that is maintained at Sandia National Labs [5]. Subsequent work [4,6,7] has been performed with Fuego, a massively parallel CFD code for predicting fire environments that was built under the Sierra architecture. Fuego is a low Mach number flow solver that uses a control-volume finite element to solve the Navier-Stokes equations together with reacting species transport equations. In 
addition, Fuego can couple the Eulerian equations of fluid motion to a Lagrangian particle tracking model. The mass, momentum and energy equations of each individual particle are updated as the particle path is tracked through the fixed fluid mesh elements. Particle drag, heat transfer, and evaporation terms contribute, respectively, to distributed source terms in the fluid momentum, enthalpy, and continuity/species equations. Droplet break-up is modeled using the Taylor Analogy Break-up (TAB) model $[8,9]$. Although droplets interact with each other indirectly in the model through the Eulerian fluid source terms, direct droplet-droplet interactions (such as coalescence) are neglected. This may be a source of inaccuracy for regions of dense droplet distribution.

This study used the hybrid TFNS turbulence model and the EDC combustion model [10], a mixing limited approximation for combustion. Presto fluid properties were similar for each case, regardless of whether fuel or water was being modeled. Standard properties for simulating water were uniformly applied. The effect of this assumption is that the density and inertia are slightly mismatched in the fuel calculation at the point of transfer. Major concepts of the spray capability in Fuego are described in DesJardin and Gritzo [9], with some updates and modifications [5, 11].

\section{Coupling Methods}

We have chosen a coupling methodology that we term one-way. The structural results are transferred to the CFD code, but there is no transfer of information from the CFD code back to the structural code. Besides being convenient, this approximation makes sense from a time scale standpoint. Typical structural code time steps are on the order of $10^{-7}$ seconds. Our CFD code is incompressible, and dynamics on the order of that scale are filtered in the formulation of the pressure and density. Fig. 1 presents a graphical illustration of typical resolution scales for the physics codes used in this study. A one-way coupling also simplifies data transfer, as the solid mechanical predictions can be run to full completion without having to wait for the comparatively slower CFD solver to predict the same interval. Because of the one-way coupling assumption, physical interactions belonging to CFD calculation are not present until the transfer to the CFD code.

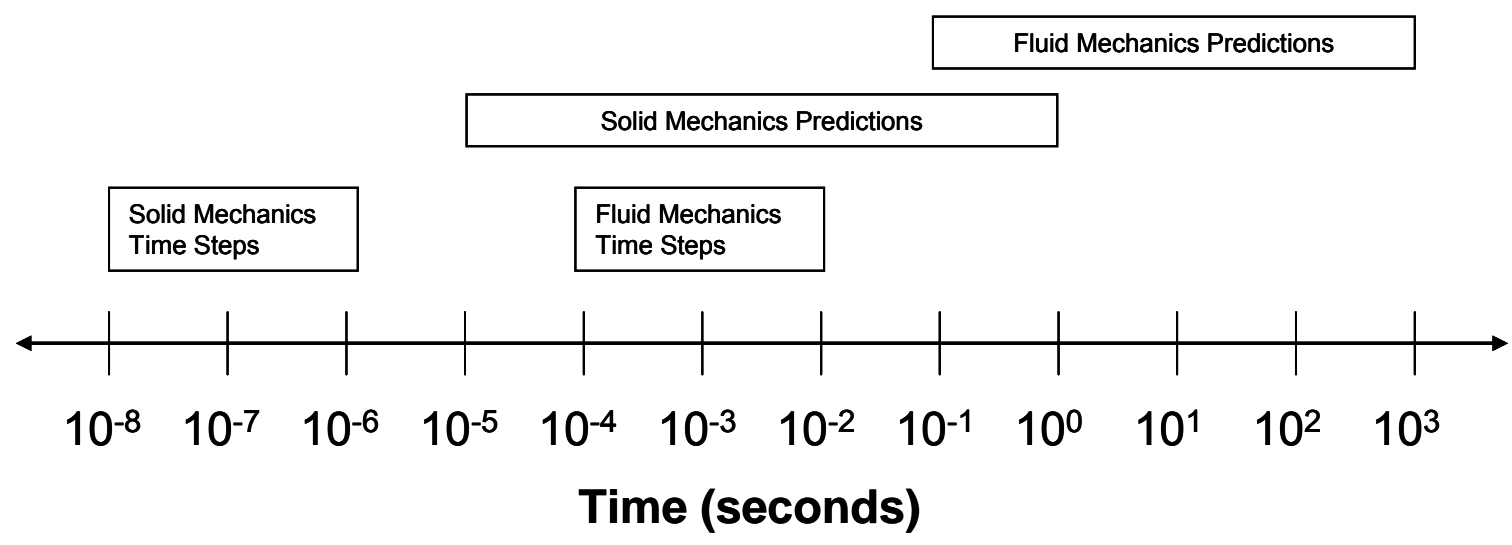

Fig. 1. An illustration of the typical time scale resolutions of the solid mechanics and fluid mechanics codes employed in this study.

A standard Presto calculation of a fluid impact uses Smoothed Particle Hydrodynamics (SPH) [12] and suitable properties for liquids to model their dispersion. Methods exist for modeling air and liquid air interactions within the SPH framework, but they are computationally intensive and cannot resolve turbulence. Further, the deformation and kinetic energy tend to dominate the dynamics in this regime, and it is usually a fair assumption to neglect the air at the time scales of the structural deformation. Much of the significant information required to appropriately begin a corresponding CFD calculation are present in the results of the structural dynamics simulation. Such key parameters include liquid position and velocity. Because of the SPH assumption, liquid morphology is not precisely known, and therefore cannot be directly extracted from the results of the model without some interpretation. The SPH approximation lends 
itself to coupling with the CFD code, which solves the equations for reacting gas flows and includes a dilute spray Lagrangian capability for modeling drops whose length scales are much smaller than that of the Eulerian mesh. With mass and momentum conservation designed into the code transfer, two of the most critical data are retained in the coupling between the codes.

An open issue of importance in the coupling is that of determining an appropriate time for the coupling to occur. In a typical structural calculation, the total kinetic energy is monitored to give an indication of the progress. When system kinetic energy changes become indiscernible or relatively small, primary structural deformations are concluding, and the physics are transitioning to a regime where the transient dynamics physics are becoming less significant. At this point other physics become comparatively more significant. The initial assumption made for these calculations was that a single time could be selected once kinetic energy changes diminished significantly and then one could transfer the mass at once into the fluid mechanics codes. This has worked successfully for some of the simulation cases. A concern with this method is that there are still dense regions of liquid fluid, and that the fluid region is abruptly disturbed by the insertions. Our CFD code employs a dilute spray approximation, and will be more stable and accurate when the liquid volume in each Eulerian cell is small compared to that of the gas. To better respect this requirement, we selected later transfer times. Recognizing that at later times the drops on the fringes of the calculation should be increasingly influenced by the air interactions, alternatives have been considered.

We have developed a staged transfer methodology that appears to improve on the problems found in the bulk transfer methods. The criterion for determining when a drop should be transferred from the structural code to the fluid code needed to be dynamic with time according to a sensible and available measure. We propose using a dimensionless separation distance, $B$, as the transfer criterion:

\section{$B=\frac{\text { CharacteristicSeparationDistance }}{\text { CharacteristicDropLength }}$}

Fig. 2 illustrates an arbitrary drop configuration and shows the variables graphically. The characteristic separation distance used has been the center to center drop distance. We have used multiple definitions of the characteristic drop length, including $2 r_{1}$ and $\left(r_{1}+r_{2}\right)$, the latter being superior for scenarios with significantly different drop sizes.

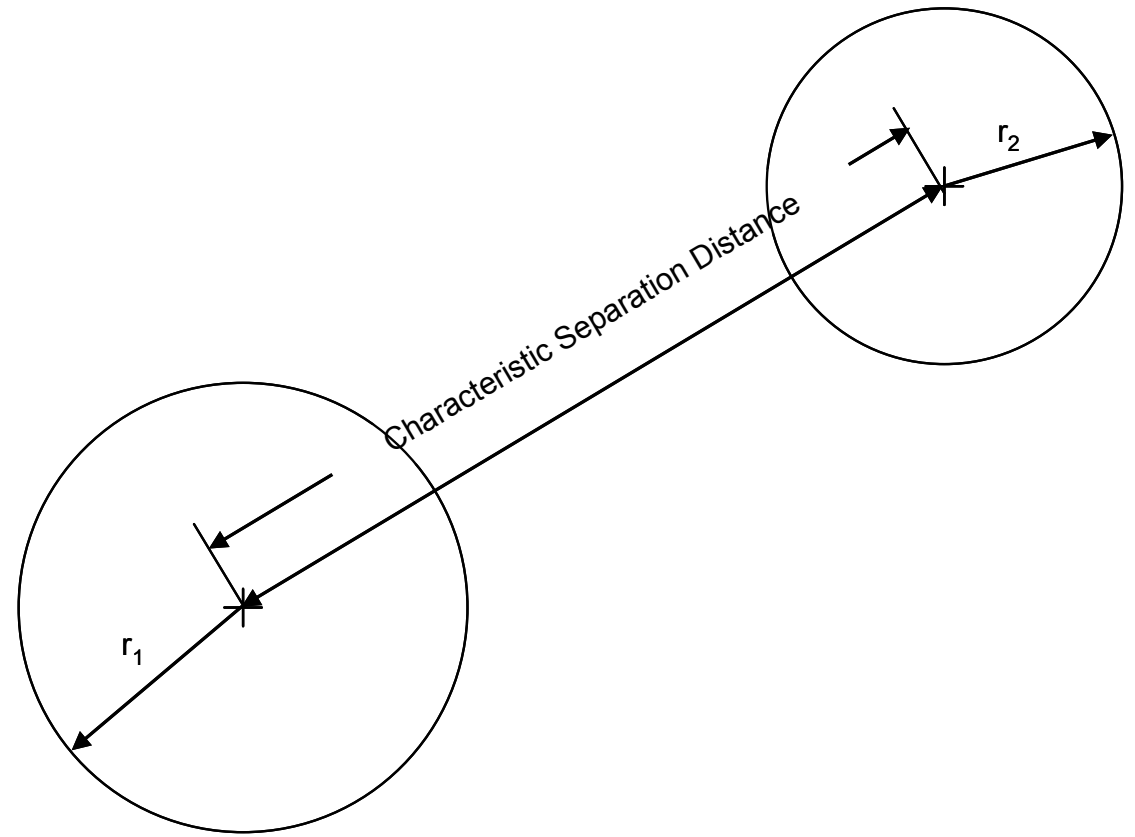

Fig. 2. An illustration of the variables used for calculating the dimensionless drop separation distance. 
To define the transfer, we extract SPH results at regular time intervals, parse the dataset for minimum dimensionless separation distances $\left(B_{\min }\right)$, and compare these values to a critical dimensionless separation distance $\left(B_{c r}\right)$. When the critical value is exceeded $\left(B_{\min }>B_{c r}\right)$, the drop is transferred to the fluid code at the time when this first occurs. The code iterates over all particles for all time steps, and a summary set of input files for the fluid mechanics simulation is created. Realistic values for $B_{c r}$ should fall between 1.0 and 2.0. At the last time step, all remaining particles are transferred. Originally, we selected 1.7 to guarantee the dilute spray, but in subsequent work we found that it may be preferable in cases to select smaller values. The value of $B_{c r}$ was treated as a parameter in two of the applications, and will be further discussed in the results section.

\section{Drop Break-up Model}

A drop break-up model is required in the CFD code to simulate the dynamics of liquid break-up with any accuracy. The model is particularly important, as the momentum transfer from the liquid drops to the gas is highly dependent on drop size. Other phenomena like evaporation and radiation also have strong dependence on the drop sizes.

The model in Fuego used for droplet break-up is the Taylor Analogy Breakup (TAB) model developed by O'Rourke and Amsden, 1987 [8]. It is based on an oscillating and distorting liquid droplet and a springmass-damper system. The surface tension is analogous to the spring restoring force and the drag force is analogous to the external force. Fluid viscosity acts as a damping force. The TAB model is formulated as a second-order differential equation for a dimensionless droplet deformation parameter $y$ :

$$
m_{d} \frac{d^{2} y}{d t^{2}}=m_{d} \frac{C_{F}}{C_{b}} \frac{\rho_{g}}{\rho_{l}} \frac{\left|u_{d}-u_{g}\right|^{2}}{r^{2}}-m_{d} \frac{C_{k} \sigma}{\rho_{l} r^{3}} y-m_{d} \frac{C_{d} \mu_{l}}{\rho_{l} r^{2}} \frac{d y}{d t}
$$

The left-hand side represents inertia; the first term on the right hand side is the forcing function (velocity differential between the drop and gas), the second the spring restoring force of the surface tension, and the third the viscous damping. The parameter $y$ is scaled such that the droplet is assumed to break up if $y$ becomes greater than one. Four constants, $C_{F}, C_{b}, C_{k}$, and $C_{d}$ are model constants which have been calibrated to values of $1 / 3,1 / 2,8$, and 5 respectively. The deformation parameter $y$ and its time derivative are stored as particle variables, and updated over each timestep $\Delta t$ using the analytical solution:

$$
y(t+\Delta t)=\frac{W e}{C}+\left\{\left(y(t)-\frac{W e}{C}\right) \cos (\omega \Delta t)+\frac{1}{\omega}\left(\dot{y}(t)+\frac{y(t)-W e / C}{\tau_{b}}\right) \sin (\omega \Delta t)\right\} \exp \left(-\Delta t / \tau_{b}\right)
$$

$$
\dot{y}(t+\Delta t)=\frac{\frac{W e}{C}-y(t)}{\tau_{b}}+\left\{\frac{1}{\omega}\left(\dot{y}(t)-\frac{y(t)-W e / C}{\tau_{b}}\right) \cos (\omega \Delta t)-(y(t)-W e / C) \sin (\omega \Delta t)\right\} \omega \exp \left(-\Delta t / \tau_{b}\right)
$$

where $\omega$ is the oscillation frequency, $\tau_{b}$ is the viscous damping time, and $W e$ is the Weber number. The constant $\mathrm{C}$ is a grouping of TAB model constants $\left(C=C_{k} C_{b} / C_{F}=12\right)$. The oscillation frequency, viscous damping time, and Weber number are given by:

$$
\begin{aligned}
\omega & =\sqrt{\frac{8 C_{k} \mu}{\rho_{l} d_{p}^{3}}-\frac{1}{\tau_{b}^{2}}} \\
\tau_{b} & =\frac{1}{2} \frac{\rho_{l} d_{p}^{2}}{C_{d} \mu_{l}}
\end{aligned}
$$


$W e=\frac{\rho_{l} d_{p}\left(u_{p}-u_{g}\right)^{2}}{\sigma}$

The deformation parameter is initialized to zero at reference time zero, which is the moment of inception of the drop. Over each timestep, it must be determined whether y can exceed unity. For small enough $\tau_{b}$, the oscillation is overdamped and no breakup occurs. Otherwise, we note that $y$ must vary in the range $W e / C \pm A$, where:

$$
A=\left((y(t)-W e / C)^{2}+(\dot{y}(t) / \omega)^{2}\right)^{1 / 2}
$$

So if $W e / C+A$ exceeds unity, we must solve for the time at which the solution to Eq. 2 becomes greater than one; if this occurs at a time less than $t+\Delta t$, the drop breaks during the interval. New drops are created in such a way as to conserve mass and energy, and as a consequence the new drop sizes fall out of the conservation equations. The new drop diameter is calculated using the following equation:

$$
d(t+\Delta t)=d(t) /\left[1+\frac{C_{k} K}{20}+\frac{\rho_{l} d_{p}(t)^{3}}{8 \sigma} \frac{6 K-5}{120} \dot{y}(t)^{2}\right]
$$

The constant $K$ is selected to be $31 / 3$. The velocities of the new drops are set equal to the original drop velocity plus a random perpendicular perturbation, with a perpendicular magnitude given by:

$$
u_{\perp}=C_{v} C_{b} r \dot{y}
$$

where $C_{v}$ is a constant calibrated to 1.0 .

A problem with the TAB model was found when using it for this coupling methodology. We are introducing drops that are moving very rapidly into a nearly quiescent environment. The drop break-up was occurring so quickly compared to reasonable fluid time steps that instability was developing in the interface and causing Eulerian divergence. This problem is aggravated when more Lagrangian sub-steps are taken for each Eulerian timestep, as is normally the case. To ameliorate this problem, we employ an additional break-up criterion: for the interval $t=0$ to $\Delta t$, break-up will not occur. In most cases, this added break-up limiter resulted in more stable calculations. It requires the assumption that the break-up process is not instantaneous, and has a magnitude on the order of $\Delta t$. For one of the test cases, the instances of this limiter reducing break-up was tracked, and found to only affect incipient drops in the first few time steps of their existence. This gave confidence in the validity of this assumption for a universal modification to the $\mathrm{TAB}$ model as originally derived.

\section{Missing Physics}

The methods described above are well suited for predicting aspects of the problem defined in the introduction. There are, however, limitations to all models. In the case of this methodology, there are approximations that must be made in the coupling space that bridge the two codes. There are also approximations in the codes themselves. These can be hard to distinguish. However, due to the difference in physical regimes, there are a large number of approximations made in the coupling regime. Consequently, there is a need to assess and understand the effect of the coupling on the outcome of the prediction.

The staged transfer ignores any interactions between the air and the impacting object prior to impact. This results in an over-prediction in the kinetic energy of the impacting objects at their point of inception in the fluid code. This is believed to be a slight discrepancy, remedied quickly when the interactions begin in the transfer. It is unclear how to validate this particular approximation. And it is not clear that there is a simple or tractable method to improve the predictions in this regard that can be readily implemented.

After impact, our codes allow us to model the liquid and solid materials separately. Thus far, we have used SPH elements for the liquid casing (i.e. the aluminum or other metals used to compose the tanks). In all reports thus far, we have focused on the liquid dispersion and ignored the dynamics of the solid material at the point of transfer between the codes. It is possible to use the same or similar methods to create solid 
particles in the fluid code that correspond to the solid material from the structural dynamics code. In fact, this has been tested previously and demonstrated as a capability. The SPH elements for solid materials tend to not completely disassociate. Solid materials are mostly found in groupings of SPH elements. The staged transfer algorithm with the dimensionless drop spacing method is therefore not appropriate. Further, once transferred to the fluid code, there is no mechanism to maintain the grouping, and there is a tendency for the particles to act independently, which is non-physical. Because the vessels were small compared to the liquid in all the test cases thus far tested, this is not thought to be a significant assumption. But not all practical cases are that way. The quality of this assumption is thought to be proportional to the amount of solid material, so there are regimes where some further development is necessary to improve this assumption.

Other aspects of the impact dynamics are also missing in the coupling capability. A list of a few additional examples highlights some of the present concerns. The surface tension is not explicitly modeled in the structural dynamics code. Gravity and drag have also been ignored. The fluid code is thought to model well the drop dynamics, but the extent to which the morphology of the liquid phase is other than drop shaped challenges the accuracy of the assumptions. A complete listing of the assumptions may appear to reflect negatively on the current capability, but it should be noted that the momentum, mass, and position of the liquid are thought to be reasonably well modeled. If one were to rank physics by importance, these are probably the most important physics that are being modeled with reasonable accuracy. The omissions might be considered secondary, as they would be expected to fall further down the list of presumed importance. This is not to imply that they are not believed to be important, rather that the approach to this problem has begun with modeling what is believed to be the most significant physics. Subsequent work will be required to identify and improve aspects of the method that contribute most significantly to the inaccuracies and uncertainties.

\section{RESULTS AND DISCUSSION}

The methods are better understood in the context of simulations that have been performed for high-speed liquid impacts. Three scenarios have thus far been simulated, the results of which are indicative of model predictive sensitivities. Selected cases have been chosen for each scenario with the intent to better understand discretization error. Assessing discretization error is complicated for these cases by the nature of the coupling. In a normal fluid simulation, discretization involves length scale resolution, typically in three dimensions. A transient problem also involves the time-scale discretization. In our coupled cases, each of these discretizations exists in each code, which can be independently varied. Furthermore, there is a discretization inherent in the coupling methodology. Normal practice involves including a few cases verifying the discretization independence by varying one or two of these independently. However, since there are a lot more points of discretization in these combined physics calculations, it is a much more significant task to fully examine the effects of each discretization approximation.

Below are summarized the results of three previous studies employing the coupling methods described above. They are brought together in this review article to highlight findings that are best understood in the context of a series of calculations. A focus is given to the findings as they relate to discretization uncertainties and coupling methods.

\section{Water Slug Test Validation}

A series of tests involved large cylindrical tanks of red-dyed water rocketed to a high speed and impacted into a concrete barricade. While the primary intent of the tests was not focused on the liquid dispersal, the tests were instrumented such that they gave relevant data to help validate this capability [13]. A more detailed description of this work is found in a different report [4].

Fig. 3 shows a photograph of the liquid spread several hundredths of a second after impact compared with a prediction of the liquid spread from simulation case 7 . There is good qualitative agreement between the experimental and simulated results. The simulation appears to resolve less detail around the fringes, but the general shape and structure of the liquid spread compares well with the tests. 
A.

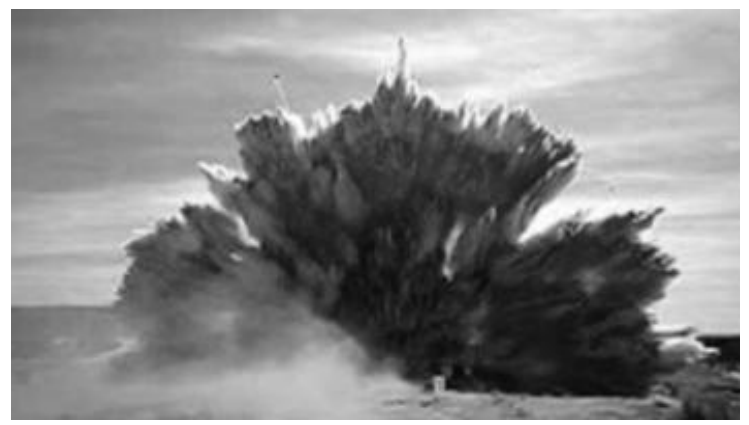

B.

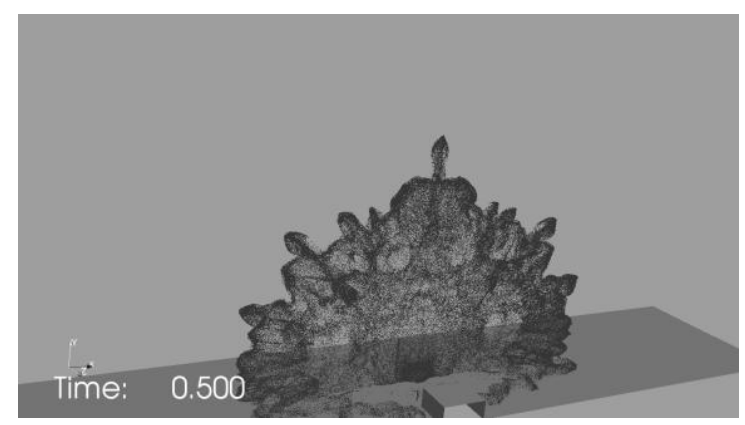

Fig. 3. Experimental (A) and predicted (B) liquid spread similar times.

The simulation test matrix is found in Table 1. The simulations did not involve any spatial discretization variations, as the baseline sizes were already quite refined. A single simulation was run with a refined fluid mesh, but this case did not achieve sufficient time to be included in the results of this study. It however verified that the results were appropriate for the short time scales.

Table 1. The water slug validation simulation test matrix.

\begin{tabular}{|c|c|c|c|}
\hline Case & $\begin{array}{c}\text { Geometry } \\
\text { Fidelity }\end{array}$ & Wind & Temporal Staging \\
\hline 1 & Low & No & No \\
\hline 2 & Low & No & 5 times $^{\mathrm{a}}$ \\
\hline 3 & High & No & 6 times $^{\mathrm{b}}$ \\
\hline 4 & Low & $2 \mathrm{~m} / \mathrm{s}$ & No \\
\hline 5 & Low & $1 \mathrm{~m} / \mathrm{s}$ & No \\
\hline 6 & High & No & 11 times $^{\mathrm{b}}$ \\
\hline 7 & High & $1 \mathrm{~m} / \mathrm{s}$ & 11 times $^{\mathrm{b}}$ \\
\hline $\begin{array}{l}\text { a Dimensionless Staging Distance: } 1.7 \\
\text { b Dimensionless Staging Distance: } 1.5\end{array}$ \\
\hline
\end{tabular}

As indicated in the test matrix, two dimensionless staging distances were assumed and four staging approximations were employed. The dimensionless staging distance used for most cases was 1.5 , with 1.7 being used for case 2. No significant differences were attributable to the change. Also, the temporal staging was not the most significant factor in the results. Results consisted of drop sizing comparisons with data, liquid spread distance, and deposition.

The geometry fidelity, which differentiated between a plain cylinder and the same cylinder with increased detail including some of the undercarriage structure, was the most significant factor in the predictions. Drop break-up was under-predicted for the low resolution geometry cases. Drop sizing predictions were generally better for cases that included temporal staging, but this evidence was weak. Higher geometry fidelity also gave qualitatively better spreading of the airborne liquid.

The deposition was analyzed both quantitatively by comparing to data and qualitatively by examining differences in the spread pattern between cases. Fig. 4 shows selected deposition results from this study, which suggests a reasonable accuracy in overall deposition when compared to the data. Of note, the local liquid deposition was mostly inaccurate, probably because a secondary splashing was not implemented for these simulations. 
A.

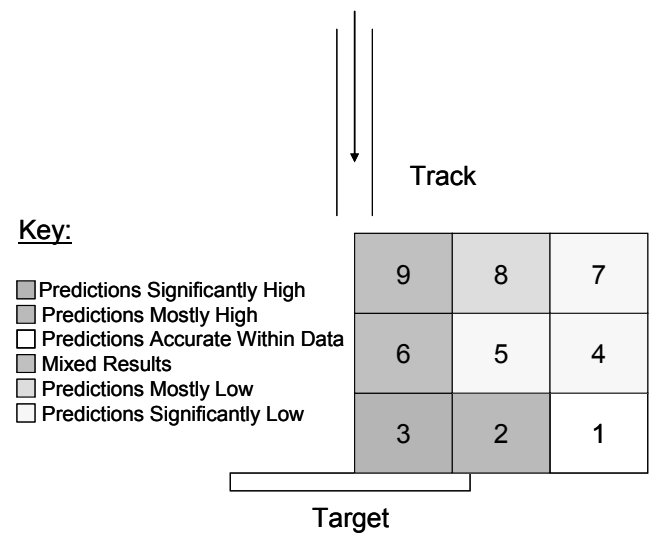

B.

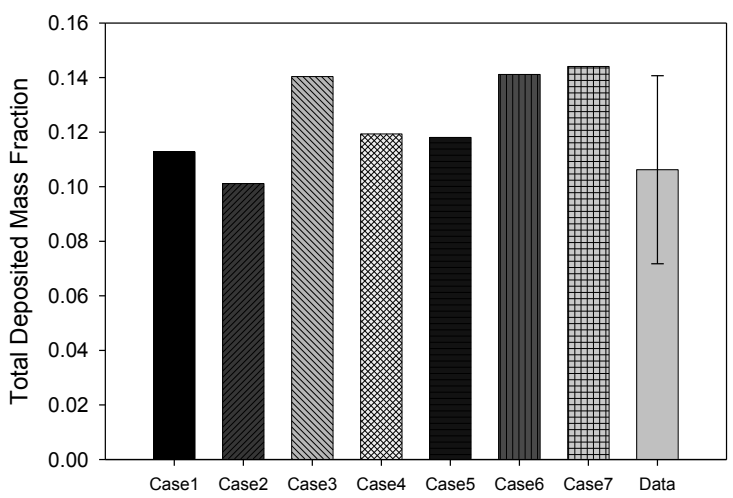

Fig. 4. Quantitative deposition results (A) of the individual pans 1 through 9, and (B) of the integrated results.

This scenario suggests the dimensionless separation distance is not a significant factor in the accuracy. Temporal staging appears to be important, and seems to be more physically realistic. It also provides confidence in the methods, as the comparisons suggest that the methodology is reasonably accurate for a physical scenario of this nature.

\section{Sled Track Test Water Brake Test Design}

In an effort to further validate this capability, we have been actively looking for appropriate data for comparisons. When large-scale tests are conducted at the Sandia National Labs rocket sled track, water is used to brake the sled to preserve the hardware for future tests. Recognizing this as a potential source for validation data, we are planning on collecting data for the spread of liquid caused by the sled braking system [7]. Water is added to a channel between the tracks, and begins to brake the sled when a customdesigned scoop ruptures the barrier of the channel at one end. In order to appropriately instrument the test, it is helpful to know approximately where points of interest are in relation to the spreading liquid and how far the liquid may spread. A limited simulation matrix was performed, as indicated in Table 2. Case F4 and F1 used the same structural dynamics predictions and varied the fluid mechanics mesh size. Case F2 and F3 used different structural dynamics predictions, case F2 examining scoop velocity and water draw variation and F3 evaluating a localized refinement within the structural dynamics calculation as suggested by Fig. 5. Fig. 6 shows an engineering rendering of the sled and a simulation result from the structural dynamics code. For all cases, a dimensionless spacing of 1.3 was used for determining mass transfer times.

Table 2. The simulation test matrix for the sled track water brake test design.

\begin{tabular}{|c|c|c|c|c|c|c|}
\hline $\begin{array}{c}\text { Fluid } \\
\text { Simulation }\end{array}$ & $\begin{array}{c}\text { Simulation } \\
\text { Transfer } \\
\text { Time (s) }\end{array}$ & $\begin{array}{c}\text { Number } \\
\text { of } \\
\text { Transfers }\end{array}$ & $\begin{array}{c}\text { Fluid Mesh } \\
\text { Elements } \\
\text { (Thousands) }\end{array}$ & $\begin{array}{c}\text { Water } \\
\text { Element } \\
\text { Size }(\mathrm{cm})\end{array}$ & $\begin{array}{c}\text { Water } \\
\text { Draw (cm) }\end{array}$ & $\begin{array}{c}\text { Initial } \\
\text { Scoop } \\
\text { Velocity } \\
\text { (m/s) }\end{array}$ \\
\hline F1 & $0.01-0.10$ & 10 & 700 & 1.9 & 10.2 & 146 \\
\hline F2 & $0.02-0.24$ & 11 & 700 & 1.9 & 15.9 & 91.4 \\
\hline F3 & $0.01-0.11$ & 11 & 700 & $0.95-1.9$ & 10.2 & 146 \\
\hline F4 & $0.01-0.10$ & 10 & 2,000 & 1.9 & 10.2 & 146 \\
\hline
\end{tabular}


A.

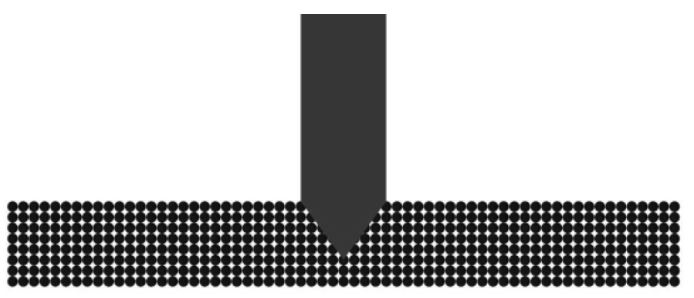

B.
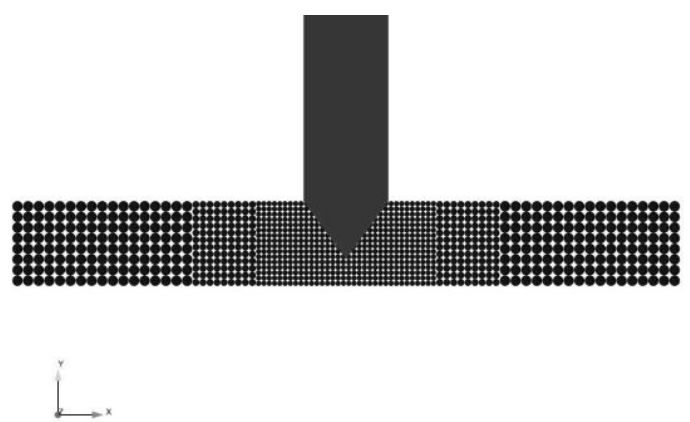

Fig. 5. A cross-section of the structural dynamics discretization for (A) simulation F1 and (B) simulation F3, the small circles representing water drops and the large rectangular arrow representing the scoop.

A.

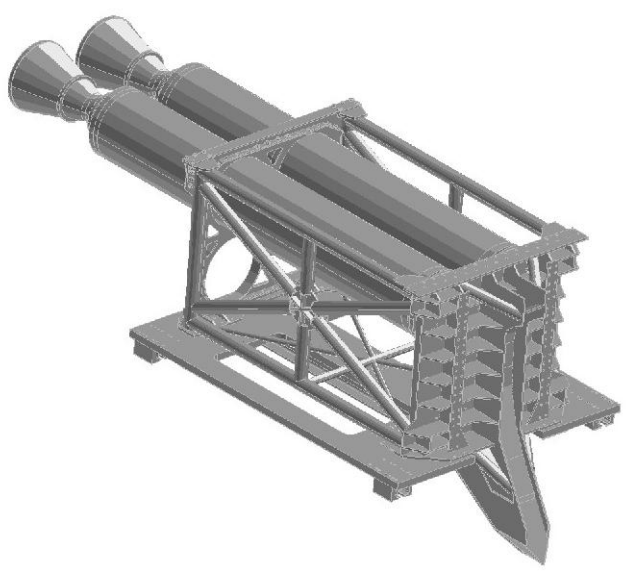

B.

Time $=0.050 \mathrm{~s}$

Fig. 6. An engineering rendering (A) of the sled and (B) a prediction result from the structural dynamics code.

Several parameters were examined in the results. Spread distances were monitored, and deposition patterns were analyzed. Gas vapor concentrations were evaluated, and drop spreading was examined. No appreciable differences were found between cases F1 and F4, suggesting adequate fluid mesh resolution for this problem. Case F2 differences from F1 were considered due to the increased liquid draw and scoop velocity. The lower velocity case with deeper draw resulted in larger deposition near the track, which suggests that better deposition data may be obtained from the slower case with a deeper pool.

Case F3 and F1 were nominally the same, with only the structural dynamics resolution changed between the two cases. The outcome was significantly different, with much lower mass deposition near the track for the case of F3. Fig. 7 shows plots of the deposition at various distances down the track from the initial impact point. The F3 case had significantly more drops exit the domain through lateral motion, and higher deposition closest to the sled track (note the difference in vertical axis scale). The magnitude of the difference is surprising and will be further investigated in subsequent work. The validation test will hopefully help improve our methods by suggesting which structural discretization yields more accurate water depositions. 
A.

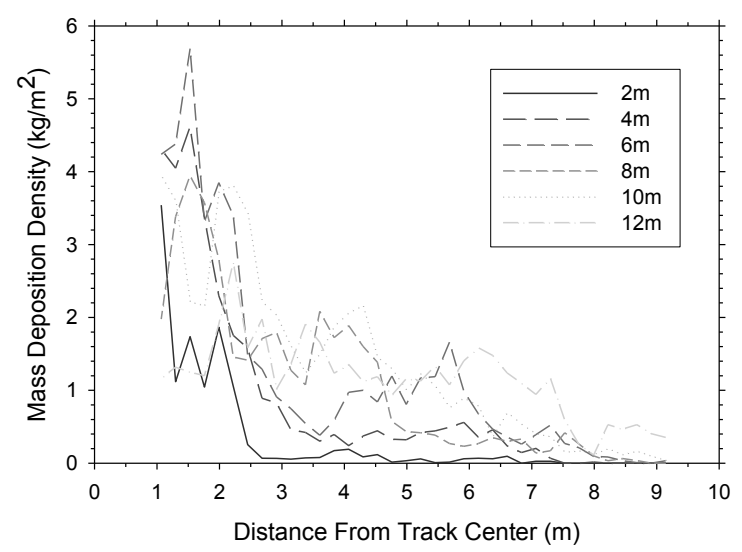

B.

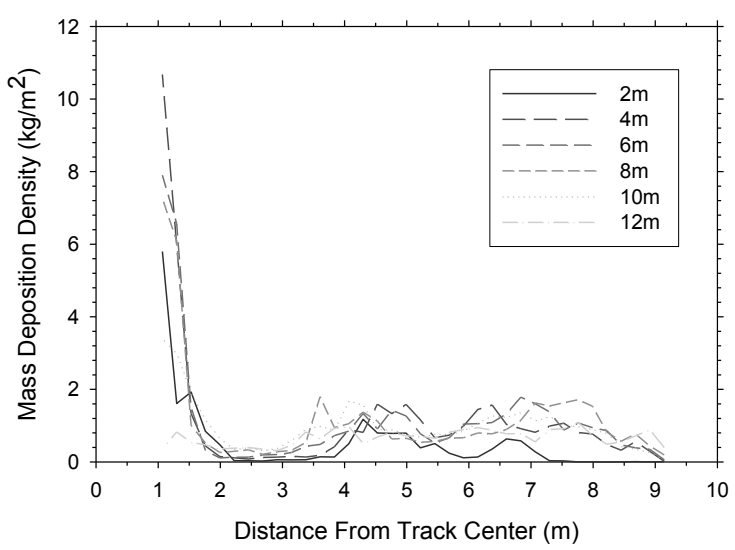

Fig, 7. Mass deposition predictions for case (A) F1 and (B) F3 at various distances down the track from the impact point.

This scenario did not suggest the fluid mesh resolution was a big factor in the predictions, but found that variable SPH sizing can give significantly different results than a coarser uniform sizing. We are attempting to perform a very large simulation with a uniformly refined structural water model to better understand this outcome.

\section{Notional Heptane Filled Cube Impact}

The most detailed discretization comparison has been done for a notional scenario of a $0.28 \mathrm{~m}$ cube with $0.0254 \mathrm{~m}$ thick aluminum walls moving at $184 \mathrm{~m} / \mathrm{s}$ into a rigid wall $[5,6]$. Two other cubes were located on either side of the impacting object. Fig. 8 shows the coarse geometry case and a rendering of the predicted fireball from case mfu. Table 3 lists the cases and their variables for this study. Because this is a notional case, it is not expected to ever have data to validate to this case, but the case comparisons are helpful for evaluating the importance of the various discretizations. Two fluid meshes, four levels of structural discretizations, four dimensionless spacings, and three temporal staging assumptions were evaluated.

A.

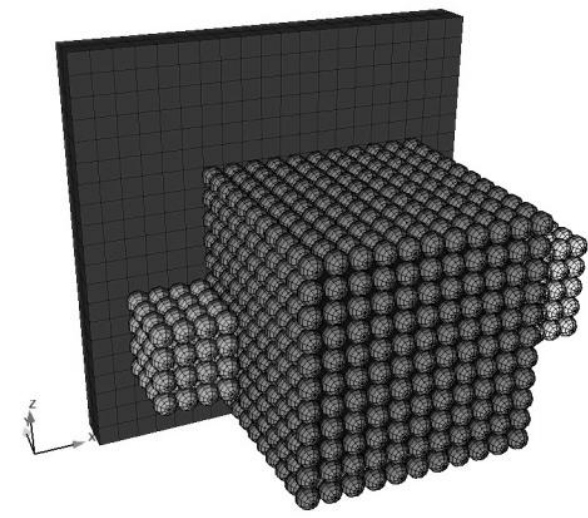

B.

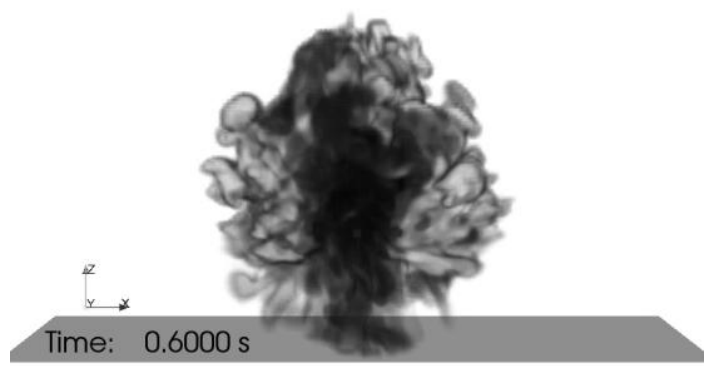

Fig. 8. Coarse geometry (A) and a rendering of the predicted fireball from case mfu (B). 
Table 3. The simulation test matrix for the heptane filled cube impact.

\begin{tabular}{|c|c|c|c|c|}
\hline Case & Fluid Mesh & Structural Mesh & Temporal Staging & Dimensionless Spacing \\
\hline $\mathrm{ccu}$ & coarse & coarse & No & \\
\hline $\mathrm{cmu}$ & coarse & medium & No & \\
\hline cfu & coarse & fine & No & \\
\hline cxfu & coarse & xfine & No & \\
\hline cfs1.1 & coarse & fine & Yes & 1.1 \\
\hline cfs1.3 & coarse & fine & Yes & 1.3 \\
\hline cfs1.5 & coarse & fine & Yes & 1.5 \\
\hline cfs1.5_18 & coarse & fine & $\mathrm{Yes}^{\mathrm{a}}$ & 1.5 \\
\hline cfs1.7 & coarse & fine & Yes & 1.7 \\
\hline $\mathrm{mfs} 1.5$ & medium & fine & Yes & 1.5 \\
\hline $\mathrm{mfu}$ & medium & fine & No & \\
\hline $\mathrm{mmu}$ & medium & medium & No & \\
\hline
\end{tabular}

All of the cases listed in Table 3 differ only in numerical assumptions, so if full convergence were attained they should all give identical predictions. They did not. The simulations are compared to each other to better understand discretization uncertainties. This helps quantify the precision of such a calculation given a range of practical discretization assumptions. This scenario is also more relevant than the previous two because it involves a reacting liquid. Results were not unrecognizably different, but there was a range of outcomes found between the cases. Fig. 9 shows prediction results of the time at which a fixed fraction $(1 / 2,1 / 4)$ of the mass remains airborne in the calculations. Fig. 10 shows the predicted number of particles.

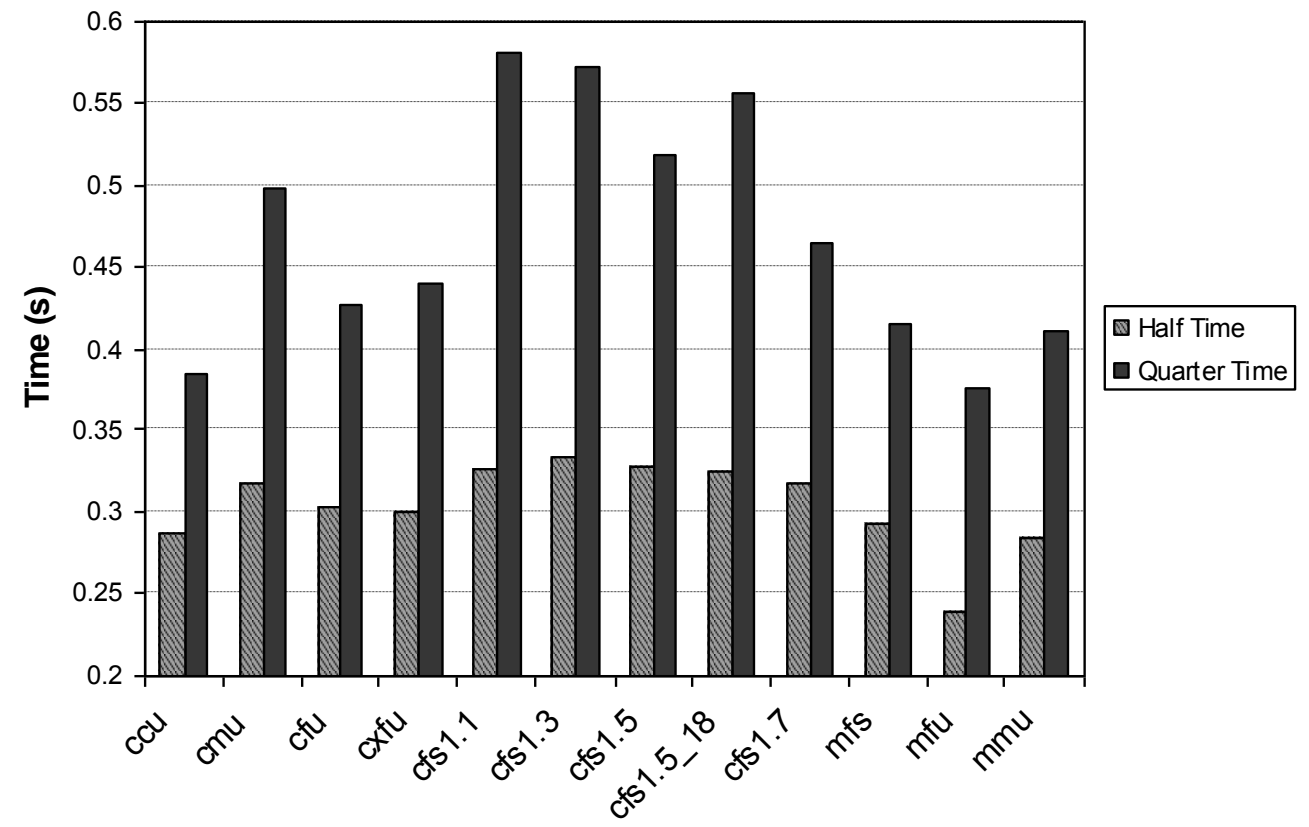

Fig. 9. Predictions of the time at which a fixed fraction of the initial liquid remains airborne. 


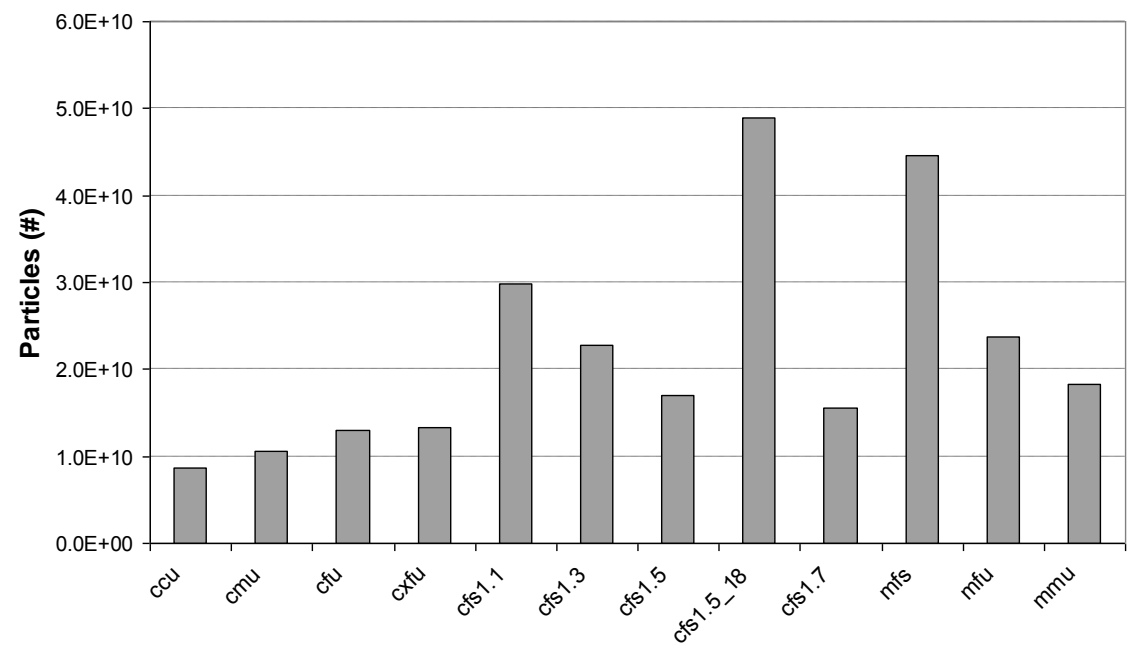

Fig. 10. Predictions of the peak number of particles predicted for each case.

Notably, there is trending in the results found in Fig. 9 and 10 as the discretization levels change. The fluid mesh in this case was found to have a significant effect on the results, generally resulting in faster evaporation and more particles. Some general trending is also found with the assumed dimensionless drop spacing. And some results trended with the structural dynamics code discretization. A surprising finding was that the cfs1.5_18 case predicted significantly different peak number of particles results. This suggests a potential need for longer transfer times and longer structural dynamics calculations to provide a wider range of times over which the transfers can take place.

\section{GENERAL DISCUSSION}

All discretization assumptions have been shown through the various scenarios to be significant to the outcome of the problem. Most of the discretization assumptions have for some cases shown to be of small significance, varying from problem to problem. There may not be a universal rule governing idealized discretization assumptions for this type of simulation, rather each problem may have a specific requirement that needs to be evaluated each time the methods are employed. This suggests that there is a need to better understand discretization limitations, and perhaps work towards developing rules or guidance for applying this methodology. Lacking better guidance, it may be best to assume the higher resolution discretization to be more accurate. The dimensionless separation distance parameter needs further evaluation to understand how it is best used for defining code coupling.

Validation is among the most immediate concerns relative to this methodology, and it is a peculiar challenge given that there are not a large number of datasets in the literature that are adequate to provide confidence in the models. Many impact tests exist in the documented literature, but they often do not involve a liquid, and less frequently document the appropriate measurements needed to validate this capability. The experiment corresponding to the sled track pre-test water dispersal event detailed in this test was performed in February of 2013, and comparisons are planned as a future endeavor. Efforts are also underway to more carefully examine the droplet size evolution, which is critical to accurate evaporation and aerosol dynamic predictions. The capability has also been examined in an impulse dispersal regime, but this regime also lacks adequate data to perform quality validation[14].

\section{CONCLUSIONS}

New methods have been developed for modeling transportation impacts and the subsequent fires. These methods have been applied successfully in three separate studies that were designed to validate methods and to understand model uncertainties. The computational uncertainty associated with the variety of time and space discretization assumptions present in the calculations has been evaluated, and shown to have a 
significant effect on the prediction results. Validation predictions performed thus far suggest the method is capable reasonably accurate predictions. Future development should pursue improved modeling methods to better model the most significant missing physics for more accurate predictions, and additional validation to help identify the most pressing needs. Lack of appropriate validation datasets suggests the need for testing to allow for better confidence in these methods.

\section{ACKNOWLEDGEMENTS}

Support of the Sandia ASC Engineering Science Research Foundation (ESRF) and ASC SIERRA programs are appreciated. Sandia is a multiprogram laboratory operated by Sandia Corporation, a Lockheed Martin Company, for the United States Department of Energy under Contract No. DE-AC04-94AL85000.

\section{REFERENCES}

[1] Edwards, H.C., "SIERRA Framework Version 3: Core Services Theory and Design," SAND report SAND2002-3616, November 2002.

[2] Gann, R.G., Editor, "Final Report on the Collapse of the World Trade Center Towers," NIST NCSTAR 1, Washington, D.C. USA, September 2005.

[3] Silde, A., S. Hostikka, and A. Kankkunen, 2011, "Experimental and numerical studies of liquid dispersal from a soft projectile impacting a wall," Nuclear Engineering and Design, 241, pp. 617 624.

[4] Brown, A.L., G.J. Wagner, and K.E. Metzinger, "Impact, Fire and Fluid Spread Code Coupling for Complex Transportation Accident Environment Simulation," Journal of Thermal Science and Engineering Applications, Vol. 4, No. 2, pp. 021004-1 to 021004-10, June 2012.

[5] Brown A.L., "Impact and Fire Modeling Considerations Employing SPH Coupling to a Dilute Spray Fire Code," Proceedings of the ASME 2009 Summer Heat Transfer Conference, ASME SHTC-2009, July 19-23, 2009, San Francisco, CA, USA, HT2009-88493.

[6] Brown A.L., "Impact and Fire Modeling for Complex Environment Simulation," The 2010 Western States Meeting of the Combustion Institute, Paper \# 10S-12, March 21-23, 2010, Boulder, CO, USA.

[7] Brown A.L., Metzinger, K.E., "Computational Test Design for High-Speed Liquid Impact and Dispersal," Submitted to the ASME/JSME 2011 8th Thermal Engineering Joint Conference, March 13-17, 2011, Honolulu, HI, USA, AJTEC-44422.

[8] O'Rourke, P. J. and A.A. Amsden, “The TAB Method for Numerical Calculation of Spray Droplet Break-up,” SAE Technical Paper 870289, 1987.

[9] DesJardin, P.E., and L.A. Gritzo, "A Dilute Spray Model for Fire Simulations: Formulation, Usage and Benchmark Problems," SAND report SAND2002-3419, October 2002.

[10] Magnussen, B. F., "On the Structure of Turbulence and a Generalized Eddy Dissipation Concept for Chemical Reactions in Turbulent Flow," 9th AIAA Sc. Meeting, St. Louis, 1981.

[11] Brown A.L., Jepsen, R.A., "An improved drop impact model for Lagrangian/Eulerian coupled codes," The ASME 2009 IMCEC Conference, November 13-19, 2009, Lake Buena Vista, FL, USA, IMECE2009-11675.

[12] Monaghan, J. J. “Smoothed particle hydrodynamics.” Rep. Prog. Phys. 68:1703-1759 (2005). http://iopscience.iop.org/0034-4885/68/8/R01

[13] Jepsen, R.A., T. O’Hern, B. Demosthenous, E. Bystrom, M. Nissen, E. Romero, and S. S. Yoon, "Diagnostics for liquid dispersion due to a high-speed impact with accident or vulnerablility assessment application,” Measurement Science and Technology, 20, 2, 025401, pp. 12, (2009).

[14] Brown, A.L., "Predictive impulse dispersal of Liquid Employing a Code Coupling Methodology," The 2013 International Seminar on Fire and Explosion Hazards, May 2013, Providence, RI, USA, 2013. 